\title{
EMBARAZO ECTÓPICO ÍSTMICO-CERVICAL EN CICATRIZ DE CESÁREA PREVIA
}

\author{
Ricardo Yáñez M. ${ }^{1}$, Pamela Martínez B. ${ }^{1}$, Yasna lbáñez D. ${ }^{1}$, Carlos Benavides B. ${ }^{1}$, Aníbal \\ Vega C. ${ }^{a}$ \\ ${ }^{1}$ Departamento de Obstetricia y Ginecología, Complejo de Salud San Borja Arriarán, Universidad de Chile.
}

a Interno, Escuela de Medicina, Universidad de Chile.

\section{RESUMEN}

El embarazo localizado dentro de una cicatriz de cesárea previa es el menos frecuente de los embarazos ectópicos y no existe un tratamiento estándar. Se presenta el caso clínico de una paciente con embarazo ectópico ístmico-cervical sobre una cicatriz de cesárea. Se hospitaliza por el riesgo de ruptura uterina, evolucionó con invasión trofoblástica de la histerorrafia culminando en muerte embrionaria. No hubo complicaciones maternas. Se trató con metotrexato para evitar la progresión de la invasión trofoblástica, con buen resultado materno.

\section{PALABRAS CLAVES: Cicatriz de cesárea, embarazo ectópico, tratamiento con metotrexato}

\section{SUMMARY}

The pregnancy within a cesarean scar is the most infrequent of ectopic pregnancy and there are no standard treatments. This is a case of a patient with ectopic pregnancy within the scar of a previous cesarean of isthmic-cervical location. She is admitted for risk of uterine rupture and with uterine scar trofoblastic invasion, ending in an embryonic death, without maternal complications. The treatment was methotrexate, avoiding trofoblastic invasion, with a favorable maternal outcome.

\section{KEY WORDS: Cesarean scar, ectopic pregnancy, methotrexate treatment}

\section{INTRODUCCIÓN}

La implantación del blastocisto en un sitio distinto al endometrio se denomina embarazo ectópico (1). Presenta una frecuencia cercana al $2 \%$ de los embarazos y es una importante causa de muerte materna $(2,3)$. Presenta distintas localizaciones, la más frecuente es en la trompa uterina $(95 \%)$ y sólo un pequeño porcentaje $(<2 \%)$ se localiza dentro de una cicatriz de cesárea previa, habiéndose reportado escaso número de casos de esta ubicación en las últimas décadas (4). Las pacientes portadoras de un embarazo ectópico en cicatriz de cesárea previa tienen elevado riesgo de presentar complicaciones tales como placenta acreta, perforación y hemorragia uterina y pérdida de fertilidad, entre otras $(4,5)$. Dado que presenta una baja frecuencia no existe consenso en cuanto al tratamiento, utilizándose actualmente medicamentos tales como metotrexato y $\mathrm{KCl}$ principalmente, además del manejo quirúrgico en casos específicos (5-7).

El objetivo de la presente comunicación es presentar y discutir el caso clínico de una paciente con embarazo ectópico en cicatriz de cesárea de localización cérvico-ístmica. 


\section{CASO CLÍNICO}

Paciente de 34 años, multípara de 1, con cicatriz de cesárea previa. Cursando embarazo de 8 semanas es derivada al Servicio de Obstetricia y Ginecología del Complejo de Salud San BorjaArriarán (CSSBA) con diagnóstico de embarazo ectópico en cicatriz de cesárea previa.

En ecografía precoz de las 5 semanas se observa esbozo de saco vitelino de $7,8 \mathrm{~mm}$ a nivel de istmo uterino, sin apreciarse estructuras embrionarias. En la semana siguiente se observa gestación ístmica en relación a cicatriz de cesárea, compatible con edad gestacional de 5,5 semanas (Figura 1). En ecografía posterior se observa saco gestacional en cicatriz de histerorrafia, con latidos cardiofetales (LCF) presentes y engrosamiento trofoblástico dentro de la cicatriz (Figura 2 A y B).

La paciente se encontraba en buen estado general, asintomática, sin metrorragia. Por su patología gestacional de alto riesgo materno se decide hospitalización para decidir conducta adecuada al caso. La paciente evoluciona clínicamente estable y asintomática. La ultrasonografía (US) muestra saco gestacional con embrión único a nivel ístmico, dentro de cicatriz de cesárea, con LCF presentes y bradicárdicos (90 latidos por minuto) (Figura 3).

Debido al importante riesgo materno-embrionario de ruptura uterina y muerte fetal, se decide realizar control ecográfico seriado para evaluar evolución de vitalidad embrionaria y decidir conducta. En nueva US se observa saco gestacional y embrión sin actividad cardiaca, por lo que se indica tratamiento con metotrexato $\left(50 \mathrm{mg} / \mathrm{m}^{2}\right.$ por vía sistémica, monodosis), para evitar mayor inva-

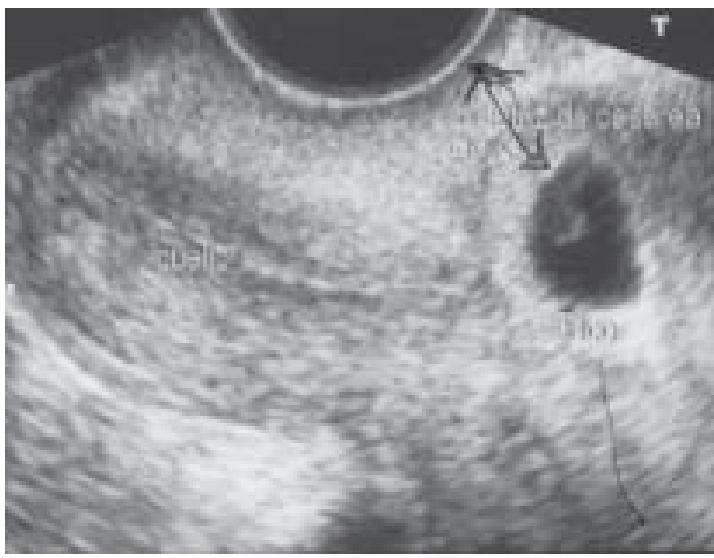

Figura 1. Se observa saco gestacional con estructura embrionaria compatible con gestación de 5,5 semanas, en porción uterina distal y en relación a cicatriz anterior.

sión trofoblástica en cicatriz uterina. Se solicita control ultrasonográfico luego de dos semanas, donde no se aprecian imágenes trofoblásticas y se observa disminución del espesor miometrial $(4 \mathrm{~mm})$ en relación a la cicatriz uterina. La paciente presentó metrorragia escasa durante este intervalo de tiempo, con buen estado general. Se continuó el seguimiento hasta el alta materna.

\section{DISCUSIÓN}

El embarazo ectópico ocurre en el $2 \%$ de los embarazos y su incidencia aumentó durante la segunda mitad del siglo XX, hasta 6 veces en los últimos 25 años $(2,3)$, y constituye una de las principales causas de muerte materna durante el primer trimestre de embarazo. En parte este incre-
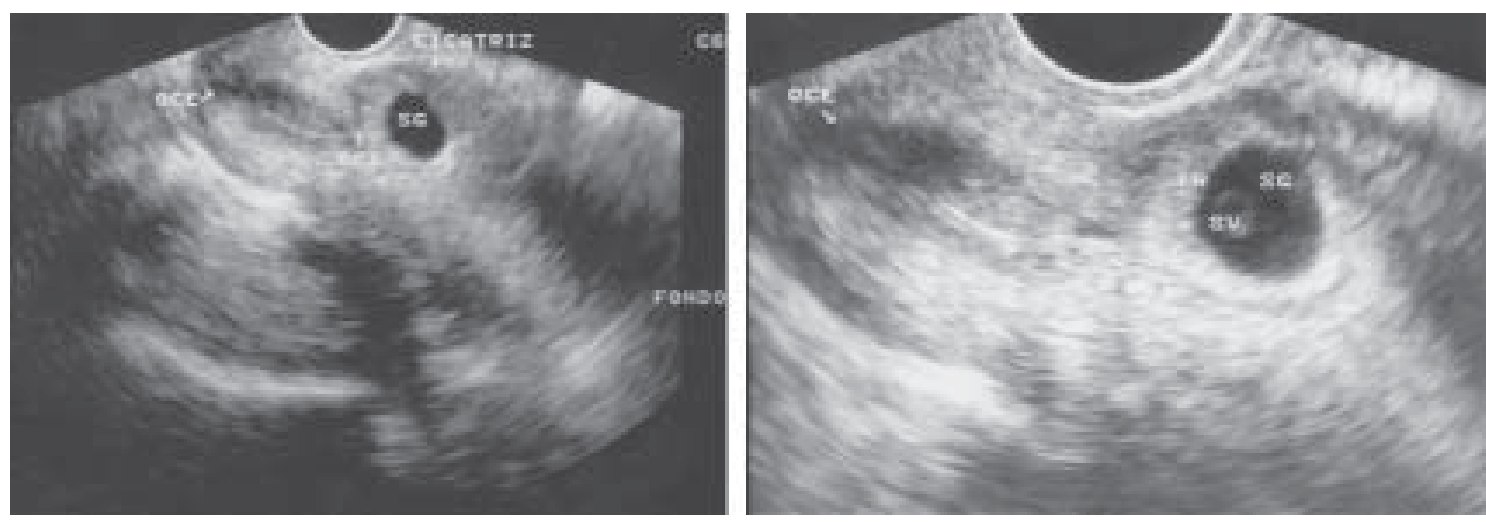

Figuras $2 A$ y $B$. Se observa saco gestacional implantado en cara anterior uterina, ocupando cicatriz de histerorrafia, con engrosamiento trofoblástico dentro de la cicatriz y a caudal en relación a orificio cervical interno (OCE=orificio cervical externo; $\mathrm{OCl}=$ orificio cervical interno; $\mathrm{SG}=$ saco gestacional; SV=saco vitelino; TR=trofoblasto). 


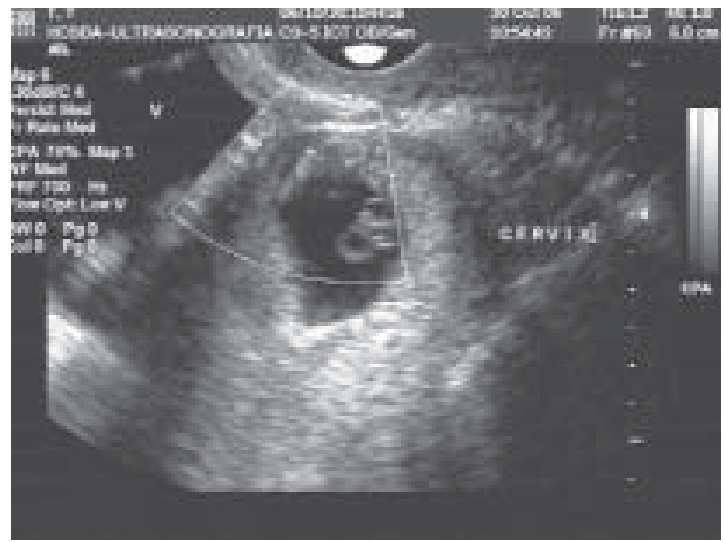

Figura 3. Se observa embarazo ectópico ístmico con invasión trofoblástica de cicatriz uterina, con LCF (+), bradicárdico, con longitud céfalo-nalgas (LCN) de $6 \mathrm{~mm}$.

mento es debido a un real aumento en la prevalencia, pero por otro lado se debe a mejores técnicas en el diagnóstico temprano de casos que de otro modo se habrían resuelto espontáneamente $(4,7)$. En el pasado cerca del $50 \%$ de las pacientes con embarazo ectópico eran ingresadas en shock e intervenidas quirúrgicamente de urgencia, realizándose el diagnóstico en ese momento $(4,6)$. Sin embargo, en la actualidad, exámenes tales como la subunidad $\beta$-HCG y la ultrasonografía de alta resolución, permiten un diagnóstico precoz y oportuno, que ha permitido variar desde una conducta netamente quirúrgica a un manejo expectante médico-quirúrgico $(5,8)$.

La mayoría de los embarazos ectópicos tiene lugar en la trompa uterina ( $>95 \%)$. En una serie de 1800 casos la distribución de los sitios fue ampular $(70 \%)$, ístmica tubaria $(12 \%)$, fimbrial $(11,1 \%)$, ovárica $(3,2 \%)$, intersticial $(2,4 \%)$ y abdominal $(1,3 \%)$ (4). Menos frecuente aún es la implantación del blastocisto dentro de la cicatriz de una cesárea previa, la historia natural de tal condición es desconocida, pero la ruptura y hemorragia de la cicatriz uterina, sobre todo en el primer trimestre, impide la continuación del embarazo, con alto riesgo de morbilidad materna y posible necesidad de histerectomía con pérdida de fertilidad futura. Por otra parte, dada la ubicación del embarazo ectópico presentado en el caso clínico existe otro riesgo, que es la invasión trofoblástica de estructuras pelvianas vecinas al útero, tales como vejiga y uréteres $(5,9)$.

La disrupción o cicatriz endometrial y miometrial pueden predisponer a una implantación anormal del blastocisto. Cuando la placenta se implanta sobre la cicatriz de una cesárea previa el riesgo de placenta acreta se incrementa significativamente (10-12). La adherencia e invasión del trofoblasto aumenta cuando la escasa decidualización del segmento uterino distal es alterada por una disrupción miometrial previa $(1,2)$.

La implantación del embarazo dentro de la cicatriz de la cesárea previa es distinto a un embarazo intrauterino con placenta acreta, la implantación dentro de la cicatriz de cesárea es una gestación completamente rodeada por miometrio y por tejido fibroso de la cicatriz y separado de la cavidad endometrial, distante a la tuba ovárica, siendo probablemente el mecanismo involucrado la invasión del miometrio a través de tractos microscópicos $(13,14)$.

La historia clínica y la ultrasonografía transvaginal pueden ayudar a diferenciar un embarazo en cicatriz de cesárea de un aborto incompleto o embarazo cérvico-ístmico, una visión ecográfica sagital a través del eje uterino longitudinal puede localizar con precisión la implantación en la cicatriz $(6,7)$.

Debido a la baja frecuencia de este tipo de embarazo ectópico no existen guías universales de tratamiento y no existe consenso en cuanto al tratamiento de elección, en reportes de casos se ha utilizado manejo médico, como lo es el metotrexato, $\mathrm{KCl}$ y solución glucosada hiperosmolar, tanto a nivel sistémico como local $(3,15-17)$. En cuanto al manejo quirúrgico se ha visto que está contraindicado el legrado uterino debido a que el tejido trofoblástico se encuentra fuera de la cavidad uterina expuesta y puede llevar a potencial ruptura de la cicatriz uterina y disrupción miometrial con hemorragia severa (18). Por lo anterior en el caso presentado se decidió el uso de metotrexato una vez que los LCF estaban ausentes para evitar la probable invasión trofoblástica del útero.

\section{CONCLUSIÓN}

El embarazo ectópico ístmico-cervical en cicatriz de cesárea anterior es una inhabitual forma de presentación de esta patología. El diagnóstico oportuno y el uso de terapia no invasiva como el metotrexato, permitió disminuir el riesgo de morbimortalidad materna, como también, preservar la fertilidad futura $(19,20)$.

\section{BIBLIOGRAFÍA}

1. Kamwendo F, Forslin L, Bodin L, Danielsson D. Epidemiology of ectopic pregnancy during a 28 year 
period and the role of pelvic inflammatory disease. Sex Transm Infect 2000;76(1):28-32.

2. Goldner TE, Lawson HW, Xia Z, et al. Surveillance for ectopic pregnancy -United States, 1970-1989. MMWR CDC Surveill Summ 1993;42:73-85.

3. Carr RJ, Evans P. Ectopic pregnancy. Prim Care 2000;27:169-83.

4. Bouyer J, Coste J, Fernandez H, Pouly JL, JobSpira N. Sites of ectopic pregnancy: a 10 year population-based study of 1800 cases. Hum Reprod 2002;17(12):3224-30

5. Fylstra DL. Ectopic pregnancy within a cesarean scar: a review. Obstet Gynecol Surv 2002;57(8):53743.

6. Taylor KJ, Meyer WR. New techniques in the diagnosis of ectopic pregnancy. Obstet Gynecol Clin North Am 1991;18:39-54.

7. Aboud E. A five-year review of ectopic pregnancy. Clin Exp Obstet Gynecol 1997;24:127-9.

8. Centers for Disease Control and Prevention. Ectopic pregnancy-United States, 1990-1992. MMWR Morb Mortal Wkly Rep 1995;44:46-8.

9. Briceño C, Alaña F, Briceño L, et al. Embarazo ístmico-cervical con invasión a región vésico-uterina. Rev Obstet Ginecol Venez 2002;62(2):123-31.

10. Miller DA, Chollet JA, Goodwin TM. Clinical risk factors for placenta previa- placenta accreta. Am J Obstet Gynecol 1997;177:210-4.

11. Chattopadhyay SK, Kharif H, Sherbeeni MM. Placenta praevia and accreta after caesarean section. Eur J Obstet Gynecol Reprod Biol 1993;52:151-6.
12. Chazotte C, Cohen WR. Catastrophic complications of previous cesarean section. Am J Obstet Gynecol 1990;163:738-42.

13. McGowan L. Intramural pregnancy. JAMA 1965;192: 637-8.

14. Fait G, Goyert G, Sundareson A, et al. Intramural pregnancy with fetal survival: Case history and discussion of etiologic factors. Obstet Gynecol 1987; 70:472-4.

15. Godin PA, Bassil S, Donnez J. An ectopic pregnancy developing in a previous caesarian section scar. Fertil Steril 1997;67:398-400.

16. Ravhon A, Ben-Chetrit A, Rabinowitz $R$, et al. Successful methotrexate treatment of a viable pregnancy within a thin uterine scar. Br J Obstet Gynecol 1997;104:628-9.

17. Donnez J, Godin PA, Bassil S. Successful methotrexate of a viable pregnancy within a thin uterine scar (correspondence). Br J Obstet Gynecol 1997; 104:1216-7.

18. Veridiano NP, Lopes J, Ohm HK, et al. Placental percreta as a cause of uterine perforation during abortion. J Reprod Med 1986;31:1049-50.

19. Bueno F, Sáez J, Ocaranza M. Tratamiento médico del embarazo ectópico. Rev ChIL Obstet Ginecol 2002;67(3):173-9.

20. Troncoso J, Devoto L, Santamaria R. Tratamiento médico del embarazo ectópico: evaluación prospectiva de la fertilidad. Rev ChIL Obstet GineCOl 2002; 67(5):354-59. 